\title{
Emerging Small Cell Wireless Technologies for 5G: Architectures and Applications
}

\author{
Mostafa Zaman Chowdhury $\mathbb{D}^{1},{ }^{1}$ Takeo Fujii $\mathbb{D}{ }^{2}$, Gabriel-Miro Muntean $\left(\mathbb{D},{ }^{3}\right.$ \\ Ji-Woong Choi $\mathbb{1}^{4}{ }^{4}$ and Giuseppe Araniti $\mathbb{1}^{5}$ \\ ${ }^{1}$ Kookmin University, Seoul, Republic of Korea \\ ${ }^{2}$ University of Electro-Communications, Tokyo, Japan \\ ${ }^{3}$ Dublin City University (DCU), Dublin, Ireland \\ ${ }^{4}$ Daegu Gyeongbuk Institute of Science and Technology (DGIST), Daegu, Republic of Korea \\ ${ }^{5}$ DIIES Department, University Mediterranea of Reggio Calabria, Reggio Calabria, Italy
}

Correspondence should be addressed to Mostafa Zaman Chowdhury; mzaman@kookmin.ac.kr

Received 18 February 2018; Accepted 18 February 2018; Published 5 April 2018

Copyright (C) 2018 Mostafa Zaman Chowdhury et al. This is an open access article distributed under the Creative Commons Attribution License, which permits unrestricted use, distribution, and reproduction in any medium, provided the original work is properly cited.

5th generation (5G) is the next step of mobile telecommunication standards. Researchers all over the world are focusing on the development of $5 \mathrm{G}$ communication systems, to be fully available for the users by 2020 . This era of wireless communication will bring new exclusive network technologies and service capabilities. Small cell network technologies will be the key driver for the $5 \mathrm{G}$ communication. It is expected that $5 \mathrm{G}$ communication will consist of ultradense heterogeneous small cell networks. The use of the traditional radio frequency- (RF-) based wireless communication is being saturated to meet the demand of $5 \mathrm{G}$. Hence, researchers are searching for new spectrum for wireless communication. The optical spectrum (infrared, visible light, and ultraviolet) is considered as a promising solution for the development of future high density and high capacity optical wireless communication (OWC) networks. The OWC offers unique advantages such as huge unregulated bandwidth and inherent security. However, the limitation of OWC is the vulnerability to obstacles. Therefore, the coexistence of RF-based omnidirectional and OWC-based directional small cells will be the excellent approach for the targeted multitier ultradense heterogeneous networks in $5 \mathrm{G}$.

The motivation behind this special issue has been to solicit cutting-edge research relevant to architectures and applications of emerging small cell wireless technologies for $5 \mathrm{G}$ communication. This special issue invited papers that address such issues. Following a rigorous review process (including a second review round), 6 outstanding papers have been finally selected for inclusion in the special issue. The accepted papers cover a wide range of research subjects in the broader area of small cell wireless technologies to meet the demand of $5 \mathrm{G}$ communications systems.

The paper "Reliability-Centric Analysis of Offloaded Computation in Cooperative Wearable Applications" by A. Ometov et al. brings into perspective the challenges related to heterogeneous communications and offloaded computation operating in cases of fault-tolerant computation, computing, and caching. The authors focus on the emerging augmented reality applications that require reliable delegation of the computing and caching functionality to proximate resourcerich devices.

The paper by E. Baktash et al. entitled "Downlink Linear Precoders Based on Statistical CSI for Multicell MIMOOFDM" investigates the linear precoder design for downlink multicell multiple-input multiple-output orthogonal frequency-division multiplexing (MIMO-OFDM) systems, where base stations (BSs) coordinate to reduce the interference across space and frequency. The authors apply the random matrix theory to approximate the ergodic weighted sum rate of the system with a closed form expression.

The paper "Diversity-Multiplexing-Nulling Trade-Off Analysis of Multiuser MIMO System for Intercell Interference Coordination" by J. Kim and C. G. Kang explores 
the performance trade-off of multicell multiuser MIMO systems for achieving intercell and intracell interferencefree conditions. The authors analyze the three-dimensional diversity multiplexing-nulling trade-off among the diversity order, multiplexing order, and nulling order. They also design a precoding matrix to mitigate both intercell and intracell interference for a linear precoding-based MU-MIMO system.

The paper by J.-E. Kim et al. entitled "Interactive Smart Fashion Using User-Oriented Visible Light Communication: The Case of Modular Strapped Cuffs and Zipper Slider Types" presents the design guideline for regulating the technical implementation, design strategies, and directions of interactive LED devices. The technology and design concepts are demonstrated through a case study of an existing LED lightbased wearable interaction. They also develop a smart fashion of modular strapped cuffs and zipper slider types that can be attached and detached according to the user's preferences.

The paper "Relay Positioning Strategy for Traffic Data Collection of Multiple Unmanned Aerial Vehicles Using Hybrid Optimization Systems: A FANET-Based Case Study" by J. Jailton et al. proposes a flight path planning model, which involves a metaheuristic optimization-based approach. The proposal relied on the artificial neural networks to optimize the positioning of the relay device, so that the throughput between the other devices could be increased.

The paper by M. T. Hossan et al. entitled "A Novel Indoor Mobile Localization System Based on Optical Camera Communication" proposes a localization technique for smartphones in indoor environments. This technique can calculate the coordinates of a smartphone using existing illumination infrastructure with LEDs. The system can locate smartphones without further modification of the existing LED light infrastructure.

\title{
Acknowledgments
}

We would like to thank all the authors who submitted their excellent research articles to this special issue and all the reviewers for providing their valuable and timely feedback through the review process, which helped to improve the quality of this special issue. The work of M. Z. Chowdhury was supported by the Korea Research Fellowship Program through the National Research Foundation of Korea (NRF) funded by the Ministry of Science and ICT (2016H1D3A1938180).

\author{
Mostafa Zaman Chowdhury \\ Takeo Fujii \\ Gabriel-Miro Muntean \\ Ji-Woong Choi \\ Giuseppe Araniti
}




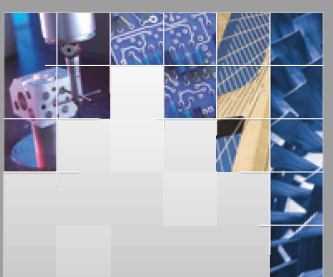

\section{Enfincering}
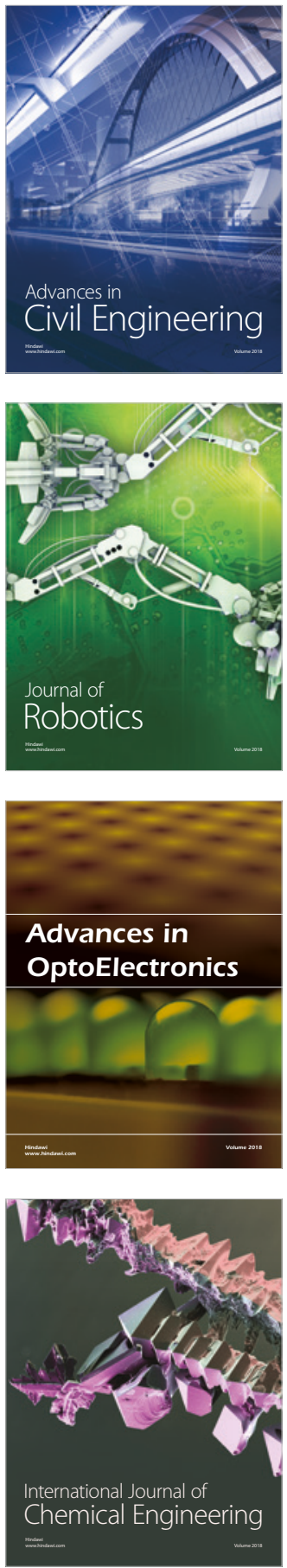

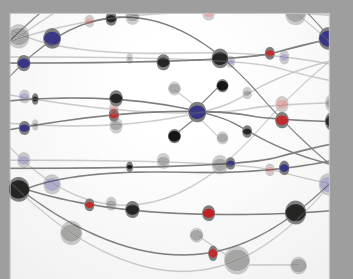

\section{Rotating \\ Machinery}

The Scientific World Journal

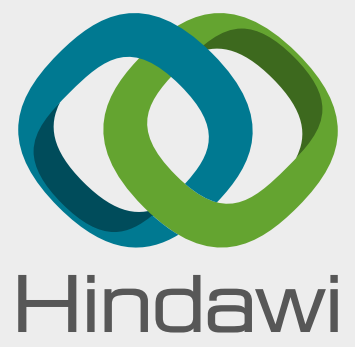

Submit your manuscripts at

www.hindawi.com
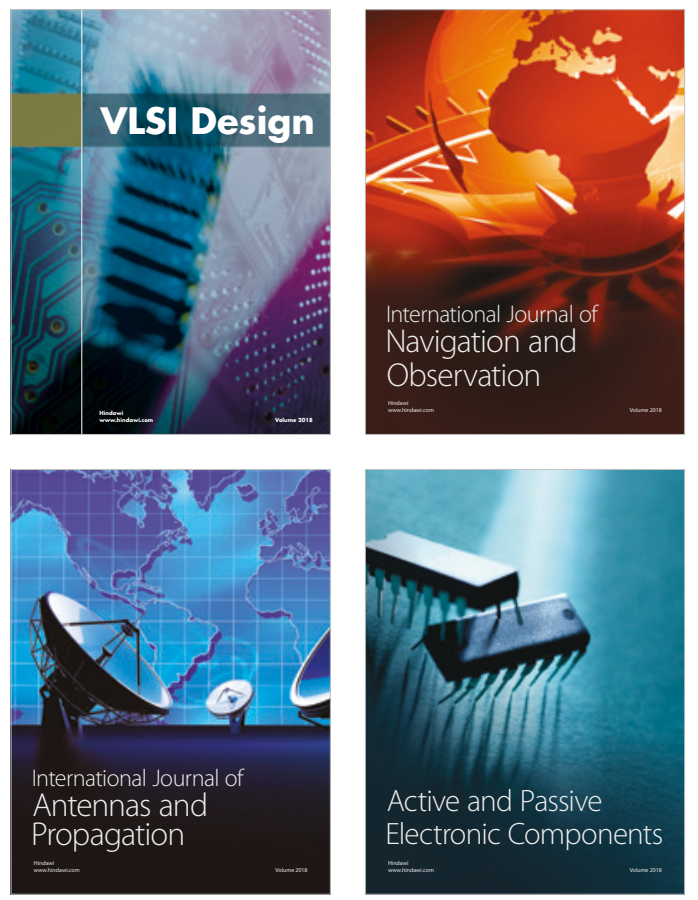
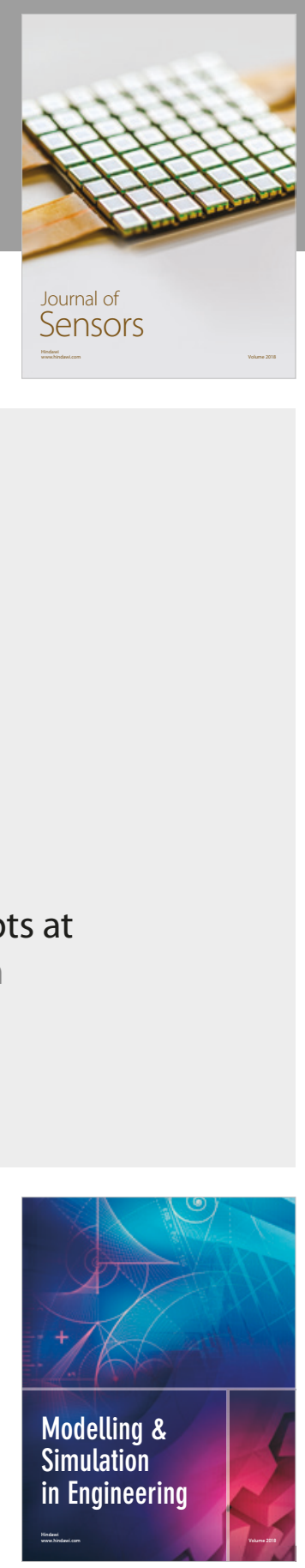

\section{Advances \\ Multimedia}
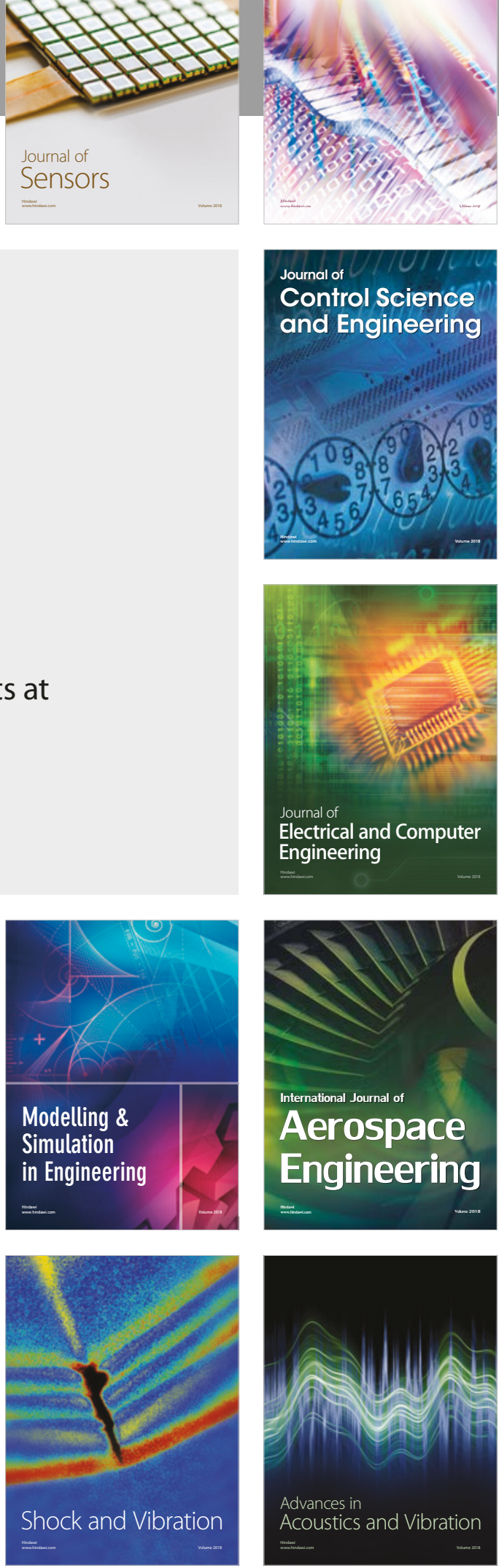